\title{
Ancora sulla distribuzione in longitudine dei flocculi di calcio
}

\author{
G. Godoli - F. Mizzzucconi - B. C. Monsignori Fossi
}

Ricevuto il 17 Aprile 1967

\begin{abstract}
Riassunto. - Viene estesa ai numeri caratteristici definitivi dei flocculi di calcio relativi agli Anni Internazionali di Quiete Solare un'analisi statistica già effettuata sui numeri caratteristici relativi all'Anno Geofisico Internazionale. La nuova analisi ha confermato l'esistenza di longitudini preferenziali. Il fuso di longitudini preferenziali per il 1965 \&े sfasato di circa $180^{\circ}$ rispetto a quello per il 1964 .
\end{abstract}

Summarx. - The statistical analysis of the definitive character figures of the $\mathrm{Ca}$ bright flocculi performed for the International Geophysical Year is extended to the International Quiet Sun Years. The new analysis has confirmed the existence of preferential longitudines. The preferential longitude belt for 1965 is out of phase $180^{\circ}$ with respect to the longitude belt for 1964 .

1. - Recentemente è stata pubblicata in questa sede (1) la lista dei numeri caratteristici definitivi dei flocculi di calcio per gli Anni Internazionali di Quiete Solare (IQ.SY, 1 Gennaio 1964-31 Dicembre 1965) la quale completa un'analoga lista per l'Anno Geofisico Internazionale (AGI, 1 Luglio 1957-31 Dicembre 1958) ( ${ }^{2}$ ).

Come già era stato fatto per il periodo $A G I\left({ }^{3}\right)$ si è ritenuto opportuno eseguire un'analisi statistica della variazione giornaliera dell'attività dei flocculi di calcio anche per il periodo IQSY. Anche per questo periodo, infatti, si è potuta nuovamente raccogliere una serie, praticamente senza lacune, di numeri caratteristici giornalieri di flocculi di calcio.

2. - L'andamento giornaliero dei numeri caratteristici dei flocculi di calcio per tutto il periodo IQSY è stato riportato nel grafieo di Fig. 1 . 
Come già è stato osservato per il periodo AGI $\left({ }^{3}\right)$, anche per il periodo IQSY si possono rilevare successioni di massimi e di minimi che si ripetono, abbastanza regolarmente, per circa 3 rotazioni sinodiche del Sole. Si è già notato $\left({ }^{3}\right)$ che questo fatto può essere attribuito alla persistenza dei più notevoli flocculi di calcio.

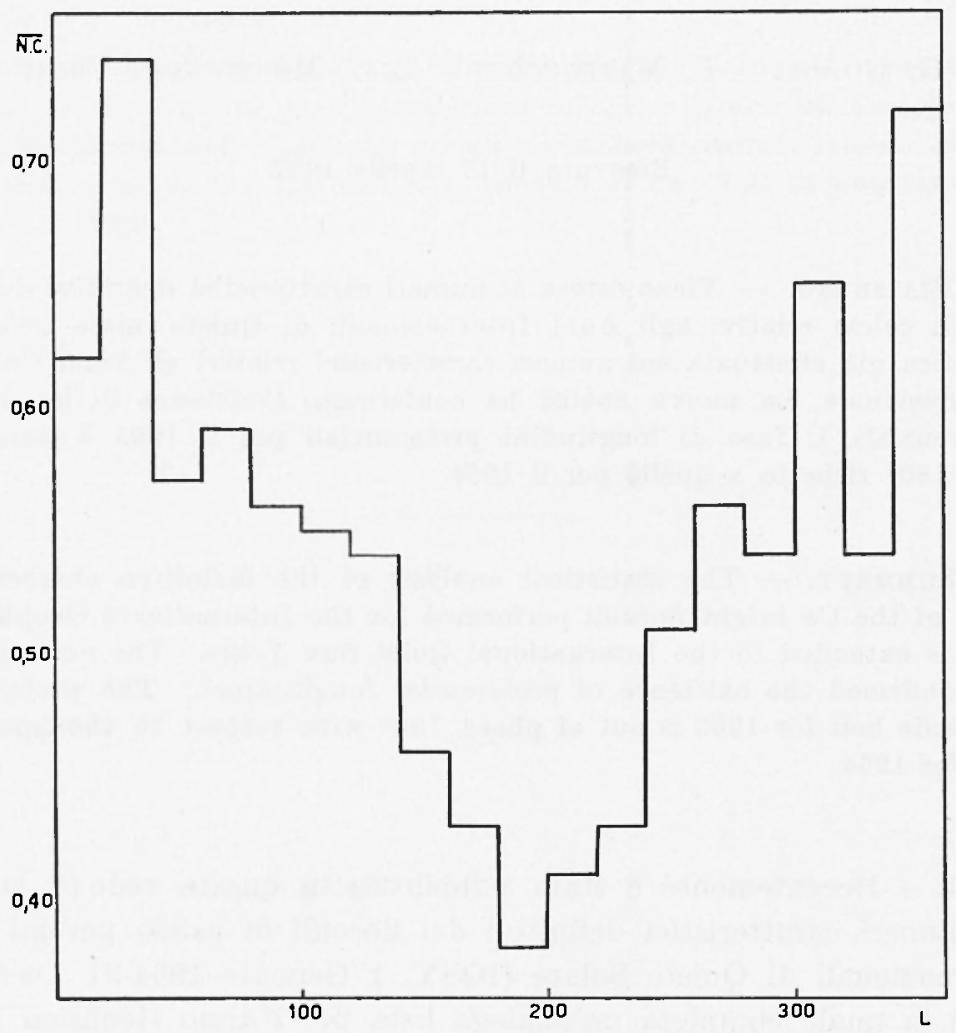

Fig. 2 - Distribuzione in classi di $20^{\circ}$ di longitudine delle medie dei numeri caratteristici dei flocculi di calcio per l'anno 1964.

Sempre analogamente a quanto è stato osservato per il periodo AGI, si può rilevare dalla Fig. 1 che le successioni di massimi tendono ad essere tra loro intervallate di multipli di 27 giorni, confermando così l'esistenza di longitudini preferenziali anche per i flocculi di calcio. 


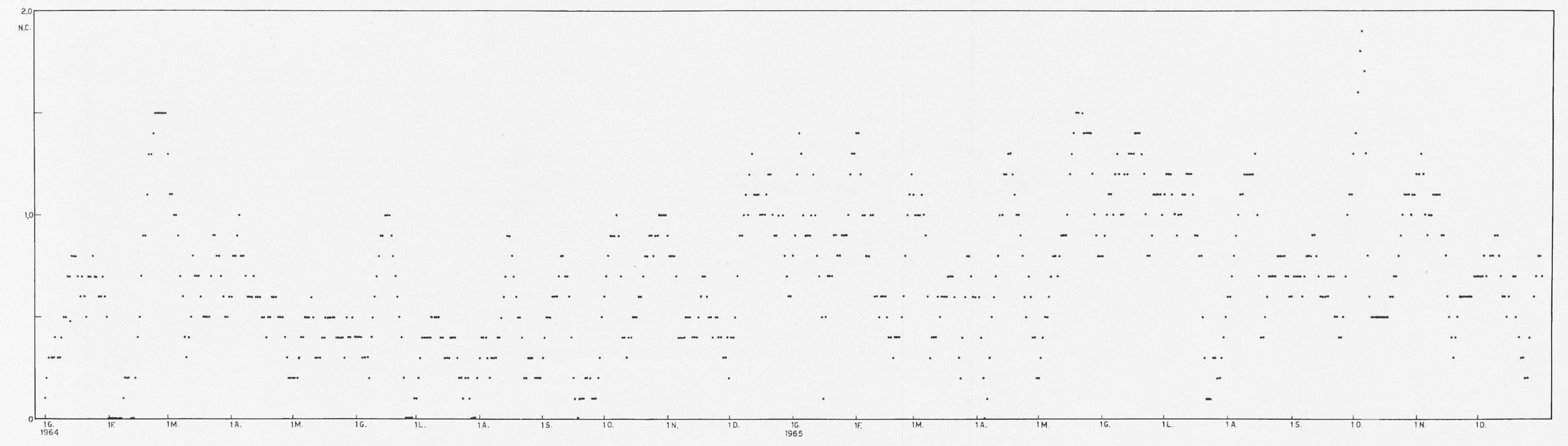

Fig. 1 - Andamento giornaliero dei numeri caratteristici dei floceuli di calcio per il periodo 1 Gennaio 1964-31 Dicembre 1965. 
Mentre però per tutto il periodo AGI il flusso di longitudini "preferenziali interessato è risultato quello compreso fra circa $300^{\circ}$ e $40^{\circ}$, per il periodo IQSY invece si sono avuti due fusi di longitudini preferenziali: per il 1964 ancora quello compreso fra circa $300^{\circ}$ e $40^{\circ}$; per il 1965 quello quasi diametralmente opposto.

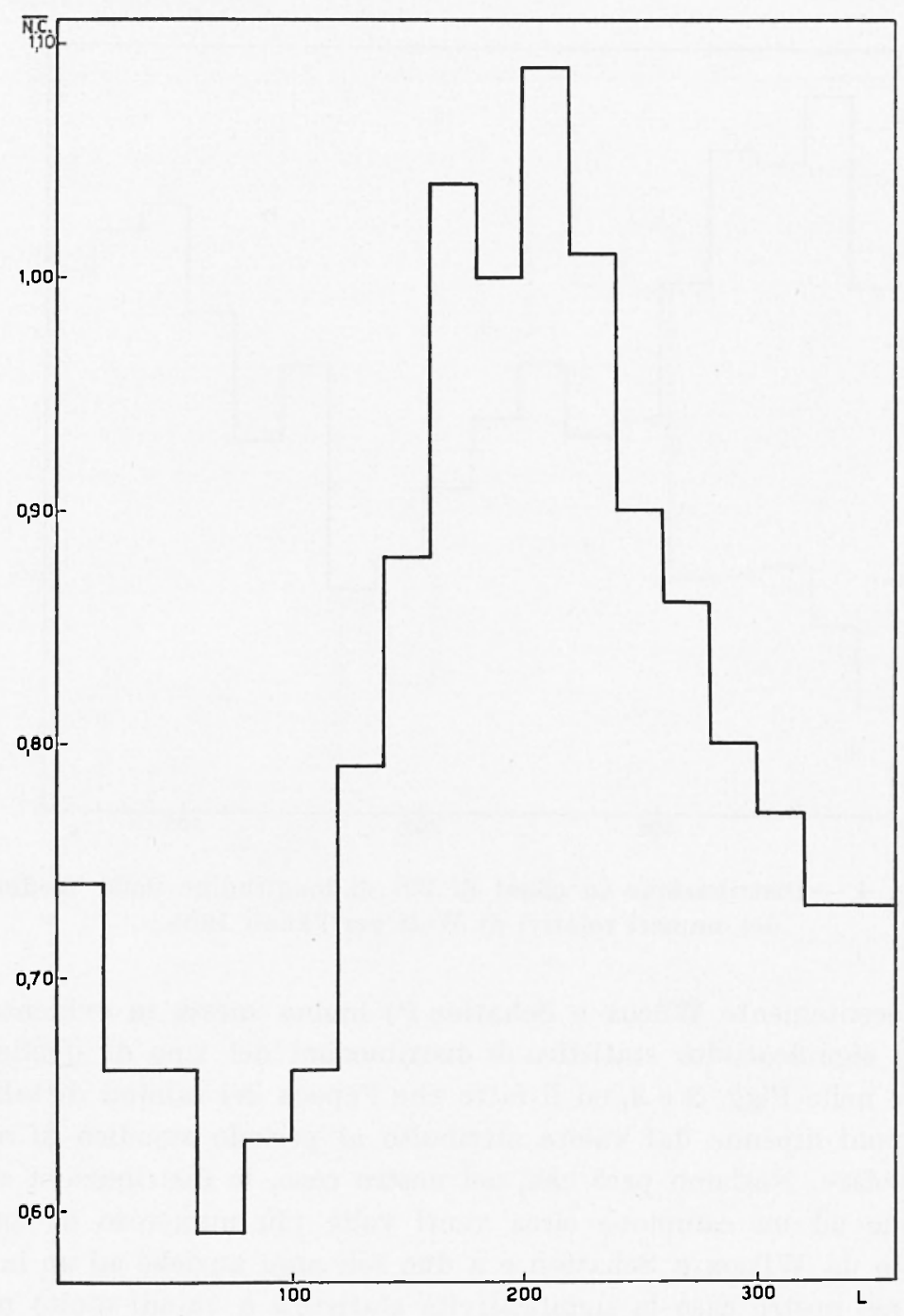

Fig. 3 - Distribuzione in classi di $20^{\circ}$ di longitudine delle medie dei numeri caratteristici dei flocculi di calcio per l'anno 1965. 
L'esistenza di fusi di longitudini preferenziali e la loro diversa localizzazione è meglio illustrata nei grafici delle Figg. 2 e 3 , in cui sono riportate separatamente per il 1964 ed il 1965 , le medie dei numeri caratteristici rilevati nei giorni in cui la longitudine del meridiano centrale era compresa nella classe di longitudine indicata in ascisse. Notiamo che ogni media si riferisce a circa venti valori giornalieri.

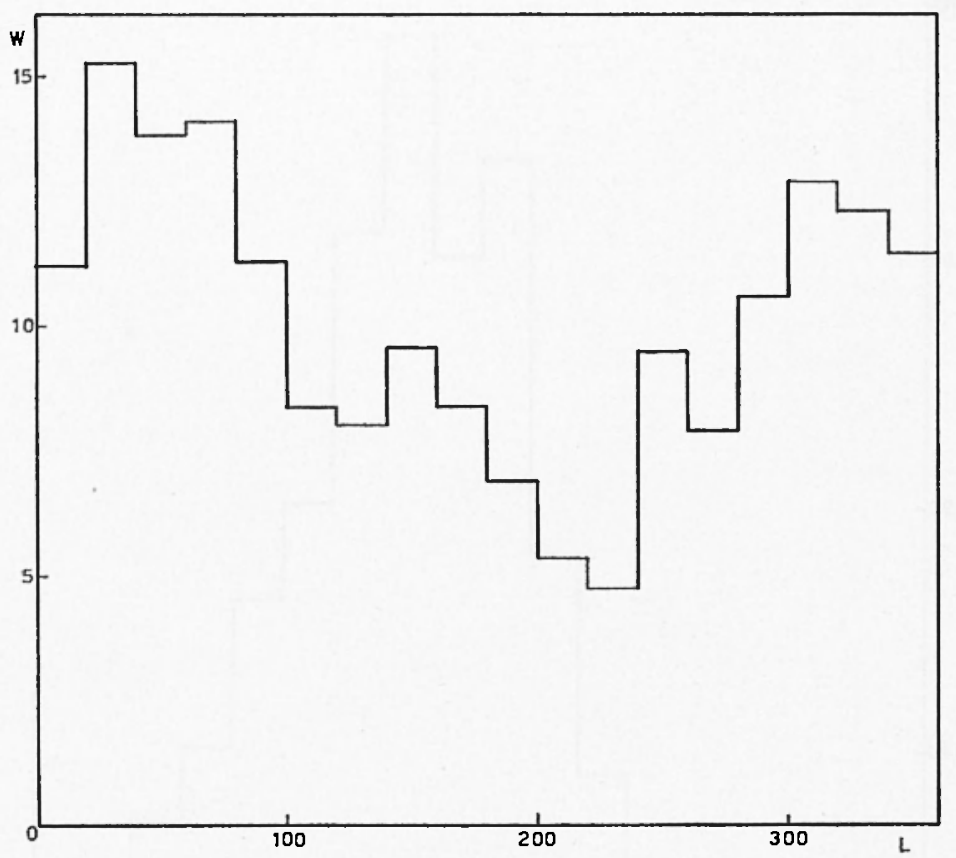

Fig. 4 - Distribuzione in classi di $20^{\circ}$ di longitudine delle medie dei numeri relativi di Wolf per l'anno 1964.

Recentemente Wilcox e Schatten $\left(^{4}\right)$ hanno messo in evidenza la piccola significatività statistica di distribuzioni del tipo di quelle riportate nelle Figg. 2 e 3 , ed il fatto che l'epoca dei minimi di tali distribuzioni dipende dal valore attribuito al periodo sinodico di rotazione solare. Notiamo però che, nel nostro caso, le distribuzioni si riferiscono ad un campione circa venti volte più numeroso di quello studiato da Wilcox e Schatten e a due soli anni anziché ad un intero ciclo; nel nostro caso la significatività statistica è quindi molto maggiore e l'influenza di errori nel periodo sinodico di rotazione solare praticamente trascurabile. 
Si potrebbe pensare che i risultati illustrati nelle Figg. 2 e 3 fossero da attribuirsi ad errori personali periodici sulla stima dei numeri caratteristici. Per questo abbiamo voluto estendere l'analisi illustratil nelle Figg. 2 e 3 anche ai numeri relativi di Wolf $(5,6)$. I risultati riportati nelle Figg. 4 e 5 confermano pienamente quelli già conseguiti per i flocculi di calcio. Sembra soltanto che per le macchie il fenomeno sia meno accentuato.

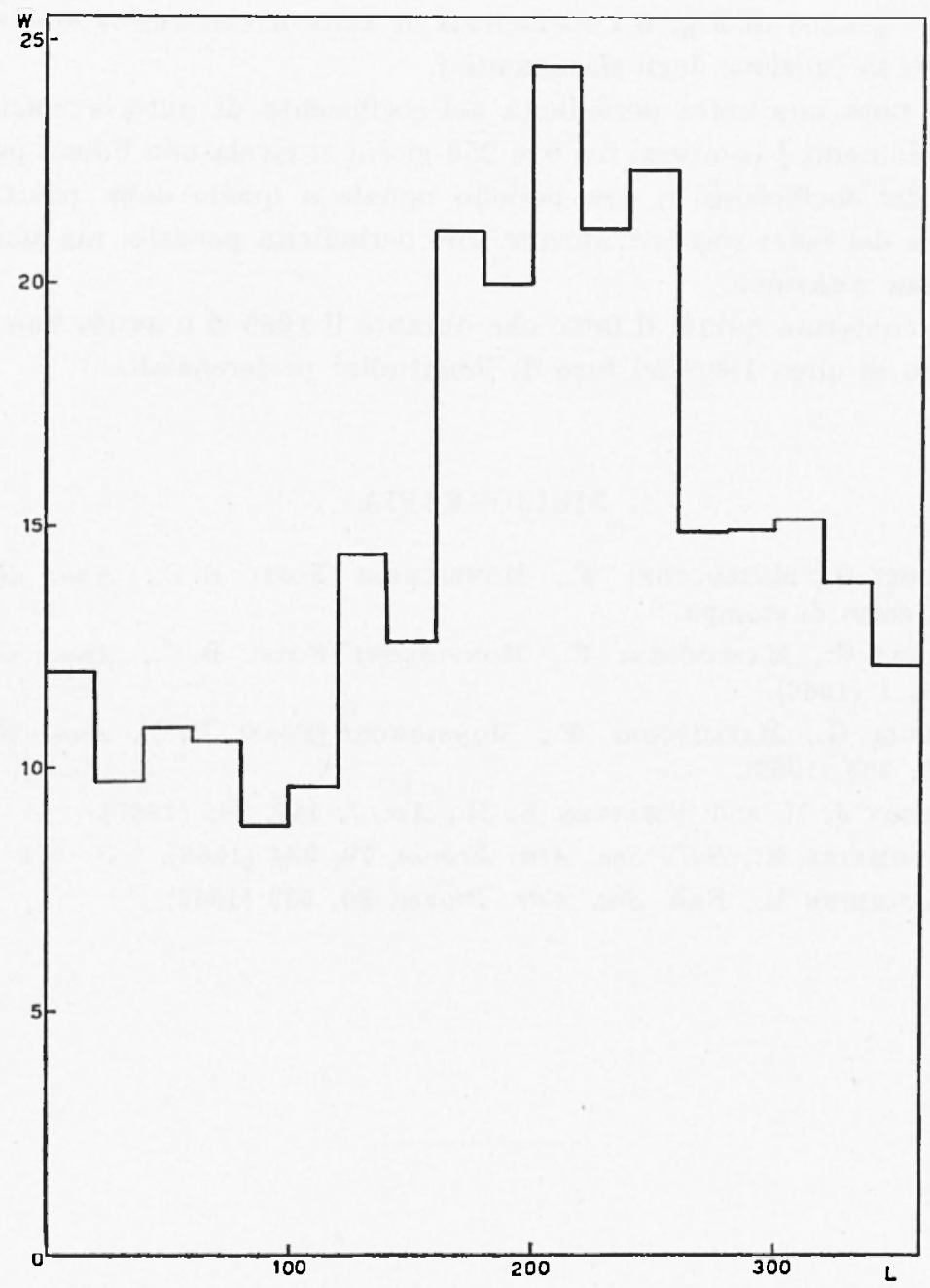

Fig. 5 - Distribuzione in classi di $20^{\circ}$ di longitudine delle medie dei numeri relativi di Wolf per l'anno 1965. 
3. - Per una più accurata analisi statistica del materiale di osservazione abbiamo calcolato, analogamente a quanto è stato fatto per il periodo AGI, il coefficiente di autocorrelazione dei numeri caratteristici dei flocculi di calcio $(N=731)$ per sfasamenti $j$ compresi fra 0 e 550 giorni. Tale calcolo è stato eseguito per mezzo di un calcolatore IBM 1620. Il programma di autocorrelazione, eventualmente adattabile anche a calcoli di correlazione, è a disposizione dei ricercatori interessati.

Nel grafico di Fig. 6 i coefficienti di autocorrelazione $r_{j}$ sono stati riportati in funzione degli sfasamenti $j$.

Si nota una netta periodicità del coefficiente di autocorrelazione. Per sfasamenti $j$ compresi fra 0 e 250 giorni si rivela una buona periodicità del coefficiente $r_{j}$ con periodo uguale a quello della rotazione sinodica del Sole; successivamente tale periodicità persiste, ma sfasata di mezza rotazione.

Si conferma quindi il fatto che durante il 1965 si è avuto uno sfasamento di circa $180^{\circ}$ del fuso di longitudini preferenziali.

\section{BIBLIOGRAFIA}

(') Godoli G., Mazzdcconi F., Monsignori Fossi B. C., Ann. Geof., in corso di stampa.

(2) Godoli G., Mazzucconi F., Monsignori Fossi B. C., Ann. Geof., 19, 1 (1966).

(3) Godoli G., Mazzucconi F., Monsignori [Fossi B. C., Ann. Geof., 19, 395 (1966).

(4) Wilcox J. M. and Schattex K. H.. Ap. J. 147, 364 (1967).

(5) Waldmeier M., Bull. Soc. Astr. France, 79, 334 (1965).

(') Waldmeier M., Bull. Soc. Astr. France, 80, 339 (1966). 
G. Godoli - F. Mazzucconi - B. C. Monsignori Fossi

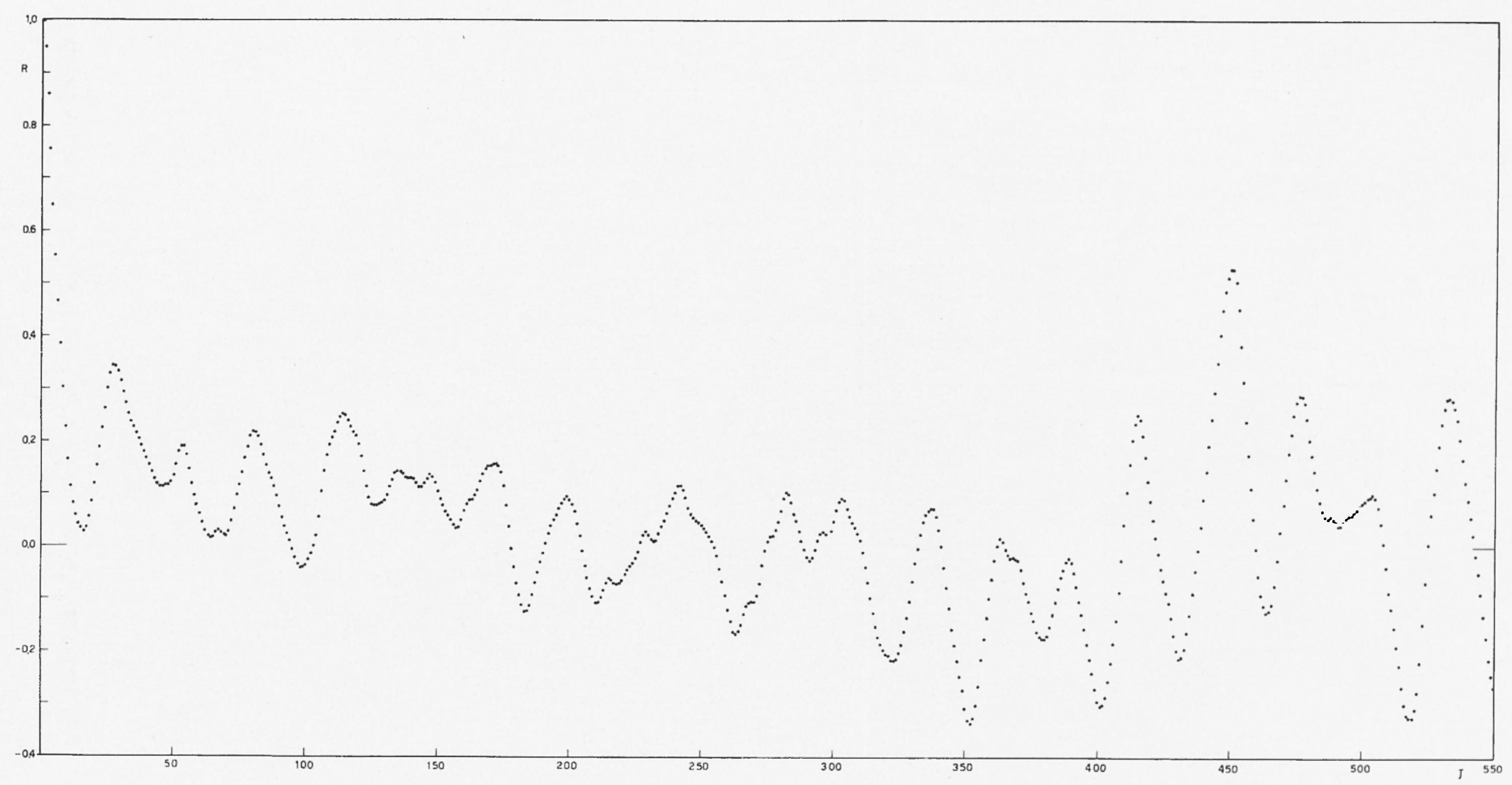

Fig. 6 - Coefficienti di autocorrelazione dei numeri caratteristici dei flocculi di calcio per sfasamenti $j$ compresi fra 0 e 550 . 